\title{
In situ revelation of a zinc-blende InN wetting layer during Stranski-Krastanov growth on GaN(0001) by molecular-beam epitaxy
}

\author{
Y. G. Cao, ${ }^{1}$ S. H. Xu, ${ }^{1}$ W. Lü, ${ }^{2}$ X. Q. Dai, ${ }^{1}$ Y. F. Chan,${ }^{3}$ N. Wang, ${ }^{3}$ Y. Liu, ${ }^{1}$ H. S. Wu, ${ }^{1}$ M. H. Xie,,${ }^{1} *$ and S. Y. Tong ${ }^{4}$ \\ ${ }^{1}$ Physics Department and the Joint Laboratory on New Materials, The University of Hong Kong, Pokfulam Road, Hong Kong, China \\ ${ }^{2}$ Institute of Physics, Chinese Academy of Sciences, Beijing 100080, China \\ and College of Materials Science and Engineering, Jilin University, Changchun 130023, China \\ ${ }^{3}$ Department of Physics, Hong Kong University of Science and Technology, Clear Water Bay, Kowloon, Hong Kong, China \\ ${ }^{4}$ Department of Physics and Materials Science, City University of Hong Kong, Tat Chee Avenue, Kowloon, Hong Kong, China \\ (Received 7 December 2004; revised manuscript received 2 February 2005; published 27 April 2005)
}

\begin{abstract}
Indium nitride ( $\mathrm{InN})$ exists in two different structural phases, the equilibrium wurtzite $(\mathrm{w})$ and the metastable zinc-blende $(\mathrm{zb})$ phases. It is of scientific interest and practical relevance to examine the crystal structure of the epifilms during growth. In this paper, we use Patterson function inversion of low-energy electron diffraction $I-V$ curves to reveal the preferential formation of zinc-blende InN wetting layer during the Stranski-Krastanov growth on $\mathrm{GaN}(0001)$. For three-dimensional islands nucleated afterwards on top of the wetting layer and for thick InN films, the equilibrium wurtzite structure is observed instead. This in situ revelation of the InN lattice structure is confirmed by ex situ transmission electron microscopy studies. Finally, the formation of zb-InN layer on $\mathrm{w}-\mathrm{GaN}$ is explained in terms of the strain in the system.
\end{abstract}

DOI: 10.1103/PhysRevB.71.155322 PACS number(s): 68.55.-a, 61.14.Hg, 68.37.Ef, 81.15.Hi

\section{INTRODUCTION}

Research in III-V nitrides continues to attract intensive attention due to their promises in optoelectronic and microelectronic applications. Recent revelations of the small energy band gap of InN have stimulated further interest in the material system, ${ }^{1-3}$ as it becomes possible that the nitridebased optical devices may cover the entire visible spectrum. Fabrication of InN quantum dots (QDs) by the StranskiKrastanov (SK) growth mode has been pursued intensively, as these QDs may be used as efficient green to near-infrared emitters. For such purposes, it is important to identify the microstructure of InN/GaN heterointerfaces, as the latter determines the electronic/optical properties of the QDs. For nitrides, an issue of particular relevance is the structural phase of the film. It is known that III-V nitrides exist in two different polymorphs - the equilibrium wurtzite $(\mathrm{w})$ and the metastable zinc-blende (zb) structures. The two differ only slightly in their formation heat $\left(\sim 21 \mathrm{meV}\right.$ per atom pair $\left.{ }^{4}\right)$, so the zb-film can be relatively easily obtained by applying constraints during epitaxy, e.g., by using a cubic substrate. ${ }^{5}$

Until recently, the structural characterization of an epilayer is mainly conducted using ex situ methods, such as $\mathrm{x}$-ray diffraction (XRD) and transmission electron microscopy (TEM). These techniques, though powerful and indispensable, suffer from certain limitations (e.g., XRD demands thick films while TEM requires sample thinning, which may lead to structural change or damage). It will thus be convenient and desirable to have an in situ method capable of revealing the microstructure of a thin film during or immediately after its growth. In this paper, we present such a method, which is based on the Patterson function inversion (PFI) of low-energy electron diffraction (LEED) $I-V$ curves. ${ }^{6,7}$ Using the method, we follow the lattice structure of epitaxial InN layer during molecular-beam epitaxy
(MBE). Interestingly, it is found that for the SK growth of InN on $\mathrm{GaN}(0001)$, the initial two-dimensional (2D) wetting layer is zinc-blende. For three-dimensional (3D) islands nucleated afterwards and for thick InN films, the equilibrium wurtzite structure is followed. We explain such a growth phenomenon in terms of the strain in the system, where the saving in strain energy by forming the zb-InN overcompensates the cost in its formation heat.

\section{EXPERIMENTS}

The MBE growth and film characterizations were conducted in a multichamber ultrahigh vacuum (UHV) system, where the MBE reactor and some surface facilities were connected via UHV interlocks. ${ }^{8}$ The MBE chamber was equipped with Knudsen cells for gallium (Ga) and indium (In) and a radio-frequency plasma unit for nitrogen $(\mathrm{N})$. The chamber contained also a reflection high-energy electron diffraction (RHEED) facility. Prior to InN growth, a thick $(\geqslant 1 \mu \mathrm{m})$ GaN buffer was grown on $6 \mathrm{H}-\mathrm{SiC}(0001)$ at $650{ }^{\circ} \mathrm{C}$. Following an annealing procedure aimed at desorbing the excess $\mathrm{Ga}$ atoms from the surface, InN deposition was initiated using an indium to $\mathrm{N}$ flux ratio of about 0.6 and a substrate temperature of $370{ }^{\circ} \mathrm{C}$. These conditions resulted in the $\mathrm{SK}$ growth mode of InN. ${ }^{9,10}$ After some specified thickness, InN deposition was stopped and the surface was quenched for characterizations in adjacent UHV chambers by scanning tunneling microscopy (STM) and the LEED. For the STM experiments, the tunneling current was $0.1 \mathrm{nA}$ and the sample bias was $-2.0 \mathrm{~V}$. In taking the LEED $I-V$ spectra, nine different incident angles ranging from $0^{\circ}$ to $39^{\circ}$ were chosen for Patterson function inversion. ${ }^{6,7}$

For separate TEM studies of the sample, a JEOL2010FEG TEM microscope was employed operating at $200 \mathrm{KV}$. The 

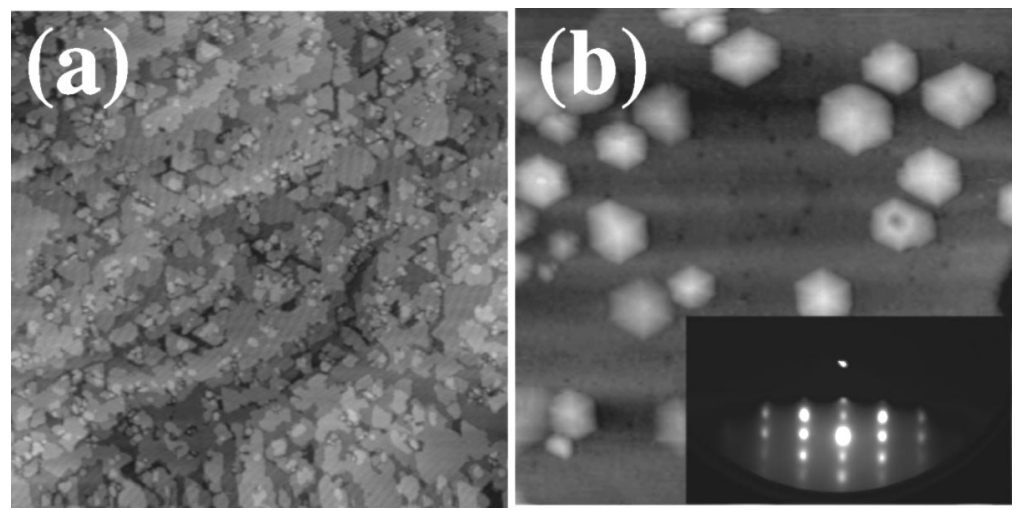

FIG. 1. STM images of InN surfaces following deposition of nominally (a) $2.5 \mathrm{BLs}$ and (b) 4.5 BLs (image size $500 \mathrm{~nm} \times 500 \mathrm{~nm}$ ). The inset in (b) shows the reflection high-energy electron diffraction pattern taken along the [11-20] azimuth.

sample was thinned mechanically and milled by argon ions according to standard procedures.

\section{RESULTS}

Figures 1(a) and 1(b) show typical STM images of surfaces following, respectively, 2.5 and 4.5 bilayers (BLs, $1 \mathrm{BL}=c / 2 \approx 2.9 \AA)$ deposition of $\mathrm{InN}$ on $\mathrm{GaN}(0001)$. In Fig. $1(\mathrm{a})$, no 3D island is observed, so the growth is $2 \mathrm{D}$ nucleation at this stage. On the other hand, by the time when 4.5 BLs material is deposited, the surface becomes populated by 3D islands as revealed in Fig. 1(b). This 2D to 3D transition is characteristic of the SK growth mode. The 3D islands are hexagonal pyramids. Closeup images of the islands reveal further that they compose double-bilayer steps at the sides and crisscross steps at the corners, which are fingerprints of wurtzite phase nitride. ${ }^{11}$ The wurtzite phase $\mathrm{InN}$ islands is also evidenced by the symmetry of the transmission RHEED diffraction spots shown in the inset of Fig. 1(b). ${ }^{12}$

To reveal the lattice structure of the 2D wetting layer, LEED $I-V$ curves were taken from the surface of Fig. 1(a). Based on these curves, PFI was conducted using the procedures as described in Ref. 6. Figure 2(a) shows the resulted PFI map from the InN wetting layer viewed in the hexagonal $(11 \overline{2} 0)$ or cubic $(10 \overline{1})$ plane. For comparison, Fig. 2(b) shows another map but derived from a thick InN film obtained by continuous deposition until all the $3 \mathrm{D}$ islands have coalesced and a continuum, smooth InN layer resulted. In these PFI maps, each dark spot represents an atomic pair in crystal. $^{6,7}$ Comparing the atomic structures of a cubic and a wurtzite layer [Fig. 2(c)], one notes that for a wurtzite film, there exist atomic pairs within a $(11 \overline{2} 0)$ plane [e.g., the ones marked by the numbers 2 and 17 in Fig. 2(c)], for which one locates vertically above the other. Such atomic pairs will give rise, in the PFI map, to a spot directly below the origin $O$. This is precisely the case in Fig. 2(b), where a strong spot $A$ is found at a distance $c=5.8 \AA$ below the origin $O$, signifying the wurtzite phase InN for the thick film. In Fig. 2(a), a similar spot $B$ is noticeable, but its intensity is much weaker than expected for a wurtzite layer. So the InN wetting layer, from which Fig. 2(a) was derived, does not seem to be wurtzite. Rather, we suspect it takes the metastable zincblende structure. Examining the atomic structure of a zb-film

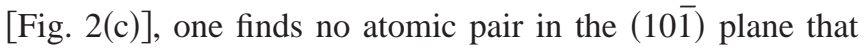

corresponds to the vertical configuration at similar distances. Hence, one expects no spot in the PFI map at location $B$ for a pure cubic film. The very existence of the spot $B$ in Fig. 2(a) then suggests either (i) the film is not of pure cubic but composed of a mixture of $\mathrm{zb}$ - and $\mathrm{w}-\mathrm{InN}$; or (ii) contributions from w-GaN buffer is non-negligible due to the thinness of the top InN wetting layer; or (iii) that there exist other structures different from cubic or wurtzite crystal that give rise to the spot. From the first sight, it is appealing to attribute the weak spot $B$ in Fig. 2(a) to the mixture of wurtzite and cubic phases of $\mathrm{InN}$ in the wetting layer, as after all, wurtzite InN represents the equilibrium structure, which can hardly be elimnated during growth. However, a close inspection of the figure shows that the spot locates at a distance $5.2 \AA$ below the origin $O$, which corresponds precisely to the lattice constant of $\mathrm{GaN}$. This observation brings doubt that it originates from the wurtzite phase $\mathrm{InN}$, or at least the proportion of $\mathrm{w}-\mathrm{InN}$ in the wetting layer is very low so as not to
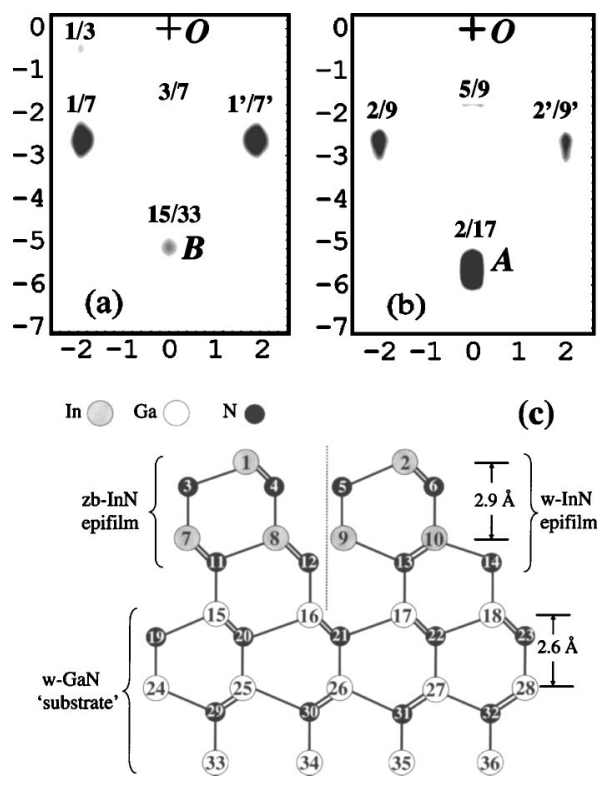

FIG. 2. LEED PFI maps of (a) InN wetting layer grown on $\mathrm{GaN}(0001)$ and (b) a thick $(\sim 300 \mathrm{~nm}) \mathrm{InN}$ film. The numbers next to the spots correspond to atomic pairs shown in (c). The two pairs labeled by $1^{\prime} / 7^{\prime}$ and $2^{\prime} / 9^{\prime}$ are, respectively, equivalent to atomic pairs $1 / 7$ and $2 / 9$ but in the second domain due to surface steps (Ref. 7). (c) Stick-and-ball model depicting wurtzite and zincblende InN epilayers on $w$-GaN "substrate." 


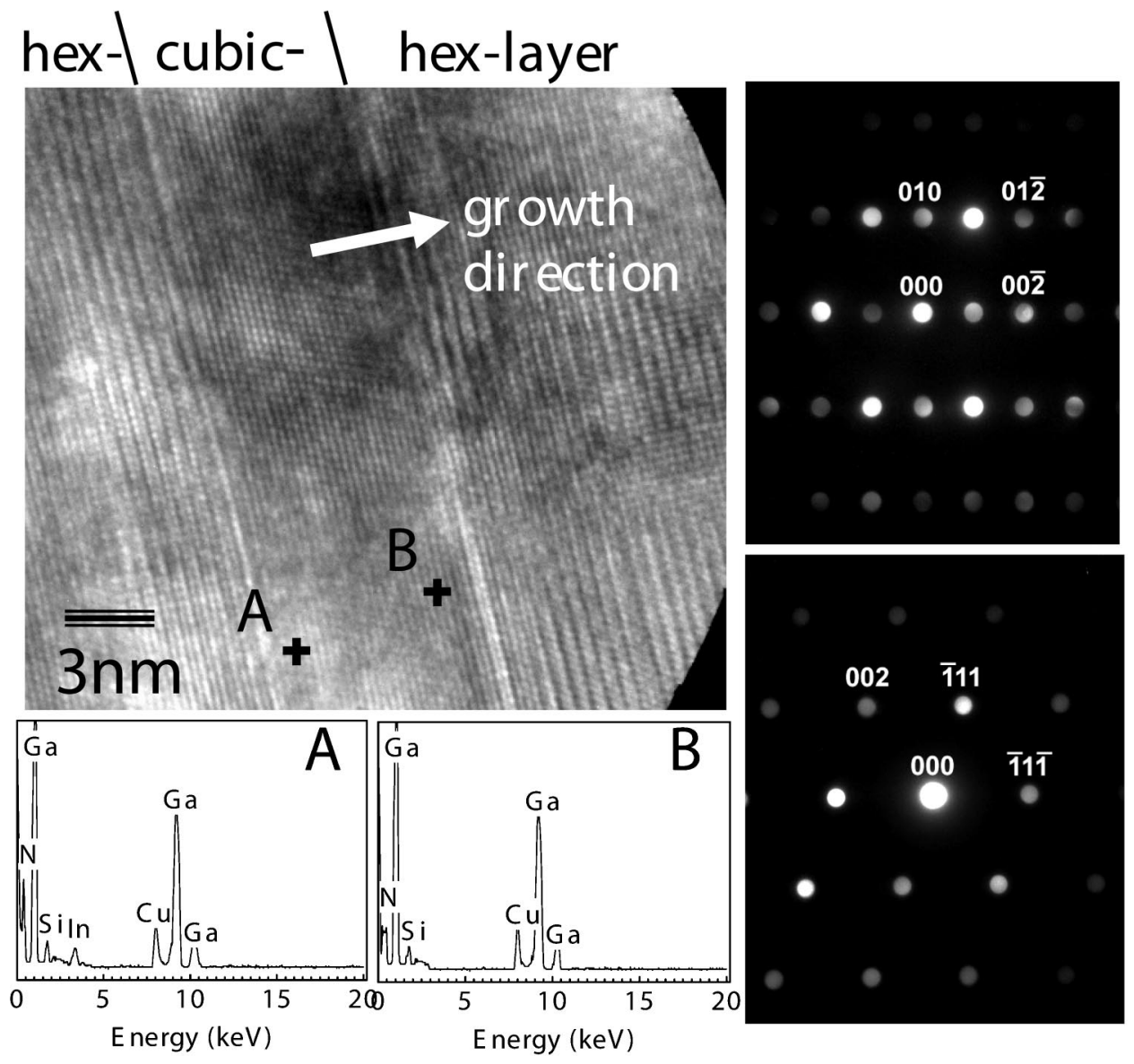

FIG. 3. High resolution TEM micrograph showing a cubic layer sandwiched between hexagonal $\mathrm{GaN}$ buffer and cap. The lower two panels show the EDX spectra taken at different spots of the middle cubic layer. The diffraction patterns shown on the righthand side are from the hexagonal capping layer (top) and the middle cubic region (bottom), respectively.

produce appreciable signal in the LEED-PFI. Indeed, if spot $B$ were due to $\mathrm{w}$-InN inclusions in the wetting layer, it would be $5.8 \AA$ below $O$ even if the layer is incommensurate to the underlying GaN, as in Fig. 2(b). On the other hand, if the layer is (partially) strained as indicated by the RHEED measurements, ${ }^{9}$ the spot would be even lower beneath $O$ (i.e., $O B>5.8 \AA$ ), as in such cases the lattice of the epitaxial InN is compressed in the plane parallel to surface but stretched in the vertical direction. This latter expectation obviously contradicts to the experimental result. So, we conclude that the spot $B$ in Fig. 2(a) is not caused by w-InN inclusions in the 2D wetting layer but originates from the wurtzite GaN buffer layer underneath [e.g., the atomic pairs $(15 / 33)$ and (16/34) in Fig. 2(c)]. Obviously, this assignment presumes that electrons scattered from the underneath layers can escape from the surface and contribute to the LEED. This assumption is validated by previous studies, which showed that atoms $\sim 10 \AA$ beneath the surface could clearly be resolved in the LEED-PFI maps. ${ }^{6,13}$ In this experiment, the nominal thickness of the wetting layer is $2.5 \mathrm{BLs}$, so contribution from the GaN buffer should indeed be observable. As for the possibility of other structures different from wurtzite and zinc-blende crystals, the one that is relevant must be related to the possible existance of surface excess metal ( $\mathrm{Ga}$ or $\mathrm{In}$ ) layers, which is known to exist if the MBE growth is carried out under the metal-rich flux conditions. ${ }^{14,15}$ The atomic arrangements of such excess metal layers have already been established. ${ }^{14-16}$ According to
Ref. 7, these layers of excess metals would produce additional spots in the PFI map, which is unseen in Fig. 2(a). Further, if the metal layers are on the surface of a cubic nitride film with the same atomic arrangement as that on a wurtzite layer, no atomic pair can yet be identified corresponding to the spot $B$ in Fig. 2(a). Therefore, we may rule out the relevance of these surface metal layers to the result of Fig. 2(a). In fact, since we have used a N-rich flux condition for InN deposition in this experiment, it is also questionable whether the excess metal layers exist.

In both Figs. 2(a) and 2(b), the other spots, such as those marked by (1/7) and (2/9), correspond to atomic pairs labeled by the same numbers in Fig. 2(c). From these spots, one may calculate the strain or the extend of alloying of the film. For example, in Fig. 2(a), one finds that the spot (2/9) locates at $(-1.92 \AA,-2.68 \AA)$, which is between the expected coordinates for pure, strain-free $\mathrm{GaN}(-1.84 \AA$, $-2.60 \AA)$ and $\operatorname{InN}(-2.05 \AA,-2.90 \AA)$. This implies that either the epitaxial layer is an InGaN alloy or the epifilm is partially strained. Since the surface layer contribution to the LEED spectra is significant, the location of the spots may also reflect the relaxation of surface atoms. Alloying is a common phenomenon during heteroepitaxy. ${ }^{17}$ However, the existence of strain in the epifilm is also evident from the RHEED. ${ }^{9}$

Finally, to verify the above finding of the PFI experiments, we prepared a sample for TEM examinations. The sample consisted of a thin (nominally $4.5 \mathrm{BLs}$ ) InN layer 
sandwiched between the GaN buffer and cap layer. The condition for depositing InN was the same as that used for growing sample Fig. 2(a), while the GaN cap layer was deposited initially at $370{ }^{\circ} \mathrm{C}$ but at $600{ }^{\circ} \mathrm{C}$ after some thickness. The purpose of using the two-temperature steps for growing the $\mathrm{GaN}$ cap layer was to preserve the structure of the underneath InN layer, and in the mean time, to maintain the crystallinity of GaN. Figure 3 shows one of the high resolution TEM images of the sample. The corresponding diffraction patterns from different areas of the sample are given on the right-hand side, and the focus mode energy dispersive x-ray (EDX) spectra are shown at the bottom. One striking feature seen in Fig. 3 is the presence of a cubic layer sandwiched between two hexagonal GaN films. The lower left hexagonal layer is the GaN buffer, while the upper right one reflects the cap layer. EDX measurements indicate, however, that the middle cubic layer contained indium only near the GaN buffer, whereas no indium was detected in the upper region. Therefore, this cubic layer composes two materials, one is In-containing nitride (InN or InGaN alloy) while the other is binary GaN. This latter result will obviously explain why the middle cubic layer is much thicker than the expected 4.5 BLs of deposited $\mathrm{InN}$. While the cubic phase $(\mathrm{In}, \mathrm{Ga}) \mathrm{N}$ is consistent with the PFI results presented above, the presence of a cubic GaN layer is, on the other hand, a bit surprising. Nevertheless, separate studies have already suggested that low deposition temperature may induce zinc-blende GaN films on hexagonal symmetrical surfaces. ${ }^{18,19}$ Indeed, the total thickness of the cubic layer in Fig. 3 corresponds rightly to that calculated from the time of the first-step, lowtemperature cap-layer deposition plus that of InN. In any case, the existence of zinc-blende (In, Ga) N at the InN/GaN heterointerface seems to be apparent from both PFI and TEM experiments.

\section{DISCUSSION}

In order to understand the preferential formation of the metastable, zinc-blende $\mathrm{InN}$ during growth on $\mathrm{GaN}(0001)$, we conducted total energy calculations examining the relative stability of zinc-blende and wurtzite InN layers pseudomorphically grown on $\mathrm{GaN}(0001)$ substrate. The calculations were based on the Vienna Ab initio Simulation Package, ${ }^{20,21}$ in which $\mathrm{Ga}(\mathrm{In}) 3 d(4 d)$ orbital were included in the valance band and their interactions with atomic cores were treated by Vanderbilt pseudopotentials. ${ }^{22}$ Supercells containing 8 BLs $\mathrm{GaN}$ plus $3 \mathrm{BLs} \mathrm{InN}$ were considered. The $\mathrm{N}$ dangling bonds at the backside of the supercell were saturated by pseudohydrogen atoms with charge $0.75 e$. The structural parameters of the GaN substrate were obtained from optimizing bulk $\mathrm{w}-\mathrm{GaN}$. Atoms in the InN layer as well as in the top bilayer $\mathrm{GaN}$ substrate were allowed to relax in the normal direction (i.e., the $c$ axis). The lateral positions of the atoms, on the other hand, were fixed at the $H 3$ or $T 4$ sites of the substrate surface, representing pseudomorphic growth of zb- or w-InN layers, respectively. Based on these calculations, it is shown that the formation energy is lowered by $14-28 \mathrm{meV}$ if the fcc stacking (i.e., the $\cdots \mathrm{ABcab}, \cdots \mathrm{ABacb}$, or $\cdots \mathrm{ABabc}$ stacking, where the capital letters refer to $\mathrm{GaN}$ substrate and the lower case letters refer to epitaxial $\mathrm{InN}$ ) is taken comparing to that of the hcp $\cdots$ ABaba stacking. Thus, a cubic $\mathrm{InN}$ layer is energetically preferred when pseudomorphically grown on GaN. Since the wurtzite phase $\mathrm{InN}$ is more stable for a relaxed film, strain must have played an important role in selecting the zinc-blende phase when grown on $\mathrm{GaN}(0001)$. In fact, the lattice mismatch between zb-InN and $\mathrm{w}-\mathrm{GaN}$ is smaller than that between $\mathrm{w}-\mathrm{InN}$ and $\mathrm{w}-\mathrm{GaN}$ (viz., 9.39-10.42\% versus $10.94-12.89 \%) .{ }^{4,23,24}$ Therefore, by forming a cubic InN wetting layer, strain energy is reduced, compared to that by growing a wurtzite layer. If this energy gain overcompensates the cost in the formation enthalpy associated with the zinc-blende structure, which is of the order of $\sim 21 \mathrm{meV},{ }^{4}$ then $\mathrm{zb}-\mathrm{InN}$ will preferentially form.

In the following, we provide a rough estimate of the elastic energy for films coherently grown on GaN. In doing so, the elastic constants calculated from first principles are used. ${ }^{24}$ Linear elasticity theory predicts that the energy due to misfit strain $f=\left(a_{\mathrm{InN}}-a_{\mathrm{GaN}}\right) / a_{\mathrm{GaN}}$ is $E_{f} \sim f^{2},{ }^{25}$ where $a_{\mathrm{InN}}$ and $a_{\mathrm{GaN}}$ are lattice parameters of (unstrained) $\mathrm{InN}$ and $\mathrm{GaN}$ films, respectively. For the given range of lattice mismatch cited above, the calculated gain in strain energy is $15-100 \mathrm{meV}$ per atomic pair, if the cubic layer is grown instead of a wurtzite layer. This is in reasonable agreement with the total energy calculations presented earlier, where the range of $35-49 \mathrm{meV}$ is suggested (i.e., the gain of $14-28 \mathrm{meV}$ in total energy plus an extra $21 \mathrm{meV}$ in formation heat).

In real situations, the $\mathrm{InN}$ layer is incoherent to $\mathrm{GaN}$ but dislocated as revealed previously. ${ }^{9,10}$ The residual strain in film is then thickness dependent. For a given thickness $H$ or a given residual strain $\epsilon$, the energies $E_{\mathrm{dis}} \sim(f-\epsilon) \ln H / H$ associated with the strain-relieving defects are different for the two polymorphs. ${ }^{25}$ Using the same elastic constants and assuming further that the strain-relieving defects are perfect dislocations, one estimates that the energy gain is about 5-30 meV for the cubic film. Note that in such cases, the energy gain will diminish gradually as the film grows thicker $(\sim \ln H / H)$. So, one may expect that the equilibrium w-InN becomes favorable for thick layers. For 3D islands, much of the strain is already relieved by both defects and elastic distortion of lattices, so the effect of strain is less significant and the wurtzite phase $\mathrm{InN}$ is obtained.

\section{CONCLUSION}

We have used the Patterson function inversion of the LEED $I-V$ curves to reveal, in situ, the structure of epitaxial InN layer grown on $\mathrm{GaN}(0001)$ by MBE. Interestingly, it is found that during its SK growth, the wetting layer is cubic, whereas for 3D islands and thick films, they are equilibrium wurtzite. This finding is supported also by ex situ TEM studies. At this stage, it is not clear whether this growth phenomenon is specific to the growth conditions used in this experiment or a more general effect. However, for the various experiments at substrate temperatures below $400{ }^{\circ} \mathrm{C}$, where the SK growth mode is known to be followed, ${ }^{9}$ and under a range of flux conditions, the cubic wetting layer has been consistently observed. The strain analysis also favors the general effect of the system. 


\section{ACKNOWLEDGMENTS}

The authors wish to thank C.-H. Lam for his useful comments and the help in strain energy analysis. Technical support from W. K. Ho is also appreciated. The project is financially supported by grants from the Research Grants Council of the Hong Kong Special Administrative Region, China (Project Nos. HKU7048/04P, 7035/03P, and CityU1238/ 02P). One of the authors (M.H.X.) also acknowledges the support by National High-Tech Research and Development (863) Program of China under Grant No. 2003AA311060.
*Corresponding author. Electronic address: mhxie@hkusua.hku.hk

${ }^{1}$ V. Y. Davydov, A. A. Klochikhin, R. P. Seisyan, V. V. Emtsev, S. V. Ivanov, F. Bechstedt, J. Furthmuller, H. Harima, A. V. Mudryi, J. Adrhold, O. Semchinova, and J. Graul, Phys. Status Solidi B 229, R1 (2002).

${ }^{2}$ J. Wu, W. Walukiewicz, K. M. Yu, J. W. Arger III, E. E. Haller, H. Lu, W. J. Schaff, Y. Saito, and Y. Nanishi, Appl. Phys. Lett. 80, 3967 (2002).

${ }^{3}$ T. Matsuoka, H. Okamoto, M. Nakao, H. Harima, and E. Kurimoto, Appl. Phys. Lett. 81, 1246 (2002).

${ }^{4}$ A. Zoroddu, F. Bernardini, P. Ruggerone, and V. Fiorentini, Phys. Rev. B 64, 045208 (2001).

${ }^{5}$ K. H. Ploog, O. Brandt, H. Yang, B. Yang, and A. Trampert, J. Vac. Sci. Technol. B 16, 2229 (1998).

${ }^{6}$ Huasheng $\mathrm{Wu}$ and S. Y. Tong, Phys. Rev. Lett. 87, 036101 (2001).

${ }^{7}$ S. H. Xu, Huasheng Wu, X. Q. Dai, W. P. Lau, L. X. Zheng, M. H. Xie, and S. Y. Tong, Phys. Rev. B 67, 125409 (2003).

${ }^{8}$ S. M. Seutter, M. H. Xie, W. K. Zhu, L. X. Zheng, H. S. Wu, and S. Y. Tong, Surf. Sci. 445, L71 (2000).

${ }^{9}$ Y. F. Ng, Y. G. Cao, M. H. Xie, X. L. Wang, and S. Y. Tong, Appl. Phys. Lett. 81, 3960 (2002).

${ }^{10}$ Y. G. Cao, M. H. Xie, Y. Liu, Y. F. Ng, H. S. Wu, and S. Y. Tong, Appl. Phys. Lett. 83, 5157 (2003).

${ }^{11}$ M. H. Xie, S. M. Seutter, W. K. Zhu, L. X. Zheng, H. S. Wu, and S. Y. Tong, Phys. Rev. Lett. 82, 2749 (1999).

${ }^{12}$ H. Okumura, K. Balakrishnan, H. Hamaguchi, T. Koizumi, S.
Chichibu, H. Nakanishi, T. Nagatomo, and S. Yoshida, J. Cryst. Growth 189/190, 364 (1998).

${ }^{13}$ H. S. Wu (unpublished).

${ }^{14}$ A. R. Smith, R. M. Feenstra, D. W. Greve, M.-S. Shin, M. Skowronski, J. Neugebauer, and J. E. Northrup, J. Vac. Sci. Technol. B 16, 2242 (1998).

${ }^{15}$ J. E. Northrup and J. Neugebauer, Phys. Rev. B 60, R8473 (1999).

${ }^{16}$ H. Chen, R. M. Feenstra, J. Northrup, J. Neugebauer, and D. W. Greve, MRS Internet J. Nitride Semicond. Res. 6, 11 (2001).

${ }^{17}$ P. B. Joyce, T. J. Krzyzewski, G. R. Bell, B. A. Joyce, and T. S. Jones, Phys. Rev. B 58, R15 981 (1998).

${ }^{18}$ M. H. Xie, L. X. Zheng, and S. Y. Tong (unpublished).

${ }^{19}$ J. W. Yang, J. N. Kuznia, Q. C. Chen, M. Asif Khan, T. George, M. De Graef, and S. Mahajan, Appl. Phys. Lett. 67, 3759 (1995).

${ }^{20}$ G. Kresse and J. Hafner, Phys. Rev. B 47, R558 (1993).

${ }^{21}$ G. Kresse and J. Furthmuller, Comput. Mater. Sci. 6, 15 (1996); Phys. Rev. B 54, 11169 (1996).

${ }^{22}$ D. Vanderbilt, Phys. Rev. B 41, R7892 (1990).

${ }^{23}$ C. Stampfl and C. G. Van de Walle, Phys. Rev. B 59, 5521 (1999).

${ }^{24}$ K. Kim, W. R. L. Lambrecht, and B. Segall, Phys. Rev. B 53, 16310 (1996).

${ }^{25}$ J. Y. Tsao, Materials Fundamentals of Molecular Beam Epitaxy (Academic, San Diego, 1993). 